\title{
An Augmentation System for Fine Manipulation
}

\author{
Rajesh Kumar ${ }^{1,3}$, Gregory D. Hager ${ }^{1,3}$,Aaron Barnes ${ }^{2,3}$ \\ Patrick Jensen $^{2,3}$, Russell H. Taylor ${ }^{1,3}$ \\ ${ }^{1}$ Department of Computer Science \\ \{rajesh, hager, rht\}@cs.jhu.edu \\ ${ }^{2}$ Wilmer Eye Institute, Johns Hopkins Medical Institutions \\ \{abarnes,psjensen\}@jhu.edu \\ Johns Hopkins University, Baltimore, Maryland, USA \\ ${ }^{3}$ NSF Engineering Research Center \\ for Computer Integrated Surgical Systems and Technology \\ Johns Hopkins University, Baltimore, Maryland, USA
}

\begin{abstract}
Augmented surgical manipulation tasks can be viewed as a sequence of smaller, simpler steps driven primarily by the surgeon's input. These steps can be abstracted as controlled interaction of the tool/end-effector with the environment. The basic research problem here is performing a sequence of control primitives. In computing terms, each of the primitives is a predefined computational routine (e.g. compliant motion or some other "macro") with initiation and termination predicates. The sequencing of these primitives depends upon user control and effects of the environmental interaction. We explore a sensor driven system to perform simple manipulation tasks. The system is composed of a core set of "safe" system states and task specific states and transitions. Using the "steady hand" robot as the experimental platform we investigate using such a system.
\end{abstract}

\section{Introduction}

Dexterous manipulation is a key element in the speed, safety, and, ultimately, the success of most surgical interventions. The majority of surgical tasks involve handheld tools operated using both vision and force (including both tactile, and kinesthetic) information. While most interventions use both force and vision at some level, the availability and efficacy of both varies widely. In general, during coarse, large-scale manipulation forces from the tools is an important cue, but visual information improves both the speed and facility of manipulation. In contrast fine, small-scale manipulation is often almost completely visual, as the interaction forces between the tool and the environment are imperceptible to even a trained surgeon. As demonstrated in the literature [1-5] [6], the lack of tactile information during surgical procedures probably results in them taking longer and being less accurate than if tactile information were present.

Our "steady hand" manipulation approach [7] requires tools to be held simultaneously by the user and the robot, with the robot complying to the forces applied to the 
tool. It provides a safe, intuitive means of addressing the above problems by augmenting the manipulation capabilities of a surgeon. It is safe, as the surgeon has direct control of the manipulator, and thereby his or her accustomed surgical tools. It is intuitive, as the surgeon not only directly manipulates those tools, but also receives direct force-feedback from the manipulator, thus "feeling" the manipulation much as one would during a large-scale surgical intervention. Our approach (compared to conventional tele-manipulation) is also more appealing because of its cost advantages.

While the increasing need for augmentation at micro scales provides a clear opportunity for human augmentation, it also makes it clear that different levels of augmentation are necessary at different stages and/or scales of surgical intervention. Open questions in the steady-hand approach (indeed, in any human-augmentation system) include 1) How might one develop a framework for human augmentation that varies its behavior in response to both the task context (e.g. scale) and the needs of the human within that context? and 2) Given such a system, does it provide "added value" to the human operator? Clearly, these two questions are closely inter-related and can only be answered through a cycle of engineering and empirical testing. In this paper, we present preliminary results based on a prototype system we have developed.

\subsection{Previous Work}

Some prior work exists in analogous problems such as automation of assembly tasks and vision guided control. Flexible automation of tasks for assembly (e.g.LozanoPerez [8], Sanderson[9]) has been studied for a long period of time. Taylor [10, 11] also looked at task representations. Some existing work in methods for learning tasks focuses on determining force/position control parameters from a human worker's operation $[12,13]$. Analysis of robot systems operating in tandem with humans and extraction of some information from this cooperation is an active topic of research. Kosuge [14-16] has looked at cooperative tasks. Exoskeletons, amplifying user input have been proposed by Kazerooni [17-19]. The vision community also has a body of research (e.g. Dickmanns [20]) in developing similar frameworks for vision-guided processes. Similar work also exists in space and planetary robotics, e.g. Lee [21] proposed a sensor-based architecture for planetary robotic sampling.

More recently, sophisticated systems have been developed to assist, or augment human actions in unstructured environments, especially in medicine. In medicine, they are often used to reduce human involvement in a task (i.e. to act as tool or camera holder) than for their superior manipulation abilities. However, Davies [22] and Troccaz [23] among others have devised systems that incorporate some level of integration of task information for constrained control. 


\section{A System for Surgical Manipulation}

The following are some of the important requirements of an augmentation system:

Safety: includes identification of critical portions of the controlled task, ability to identify and/or correct faults, and redundancy. In medical procedures, the criticality of the task puts safety as the most important design consideration.

Stability: includes meeting performance specifications over time, state/condition and over the range of inputs possible.

Efficacy/Accuracy/Functionality: includes ability to perform useful function identified by the users, and the ability to perform it without significantly modifying existing processes.

Ease of Interaction: includes ability to interact with the user with conventional tools used in the process and without imposing significant restrictions on existing practice or requiring extensive training.

Interaction with the planning process, possibility of learning/teaching, and accounting/process learning are other desirable attributes. It is difficult to design an optimal solution for tasks in such a dynamic environment and the flexibility of the system to allow tuning of it performance is likely to be important. Unlike assembly environments, augmentation must seamlessly integrate with existing processes and environment. There are other significant differences between assembly and surgical environments. Safety is critical and the environment is unstructured and very dynamic.

There are a sufficiently large number of generic manipulation actions in surgical procedures that may be augmented by a single surgical assistant. Examples of such tasks are camera holding, acting as a tool guide, tool positioning, constrained and guarded motions (force constraints are especially hard to implement without augmentation and very common in practice).

\subsection{Our Approach}

We demonstrate our approach by choosing a simple example task that involves both fine and coarse manipulation, and is a common minimally invasive task. The task of placing a tool through a port (small incision or hole) at a surgical site is composed of the following steps: $a$ ) positioning the tool at the port, $b$ ) orienting it such that it can be inserted, $c$ ) insertion of the tool, $d$ ) adjusting the orientation of the tool towards the placement site viewing through the visual feedback device (microscope in the eye, video feedback in laparascopy etc), e) approaching the site, and f) achieving contact.

This task has both coarse manipulation (positioning and orientation leading to the port) and fine manipulation inside the organ. In our approach, each step of the task is called an action. Actions are themselves more complex than a motion that can be directly coded. They are represented as chains of high-level subroutines (primitives) that are linked together by predicates. Primitives are composed of functions implementing sensing and manipulation routines. Predicates serve as conditions for transitions in the state graph composed of basic system states (for manipulation and safety), and task specific states. Predicates include both automatic sensing (e.g. contact detection) and explicit user input.(e.g. input from buttons or foot pedals ). 
Implementations of sensing and manipulation subroutines are the basic building blocks. These subroutines perform well-defined tasks that are robust to errors. Manipulation subroutines such as move to specified position, move relative distance, move with specified velocity, and sensing subroutines such as get current value, filter raw values, get biased value, resolve values with respect to a given Cartesian frame are examples of basic routines used here.
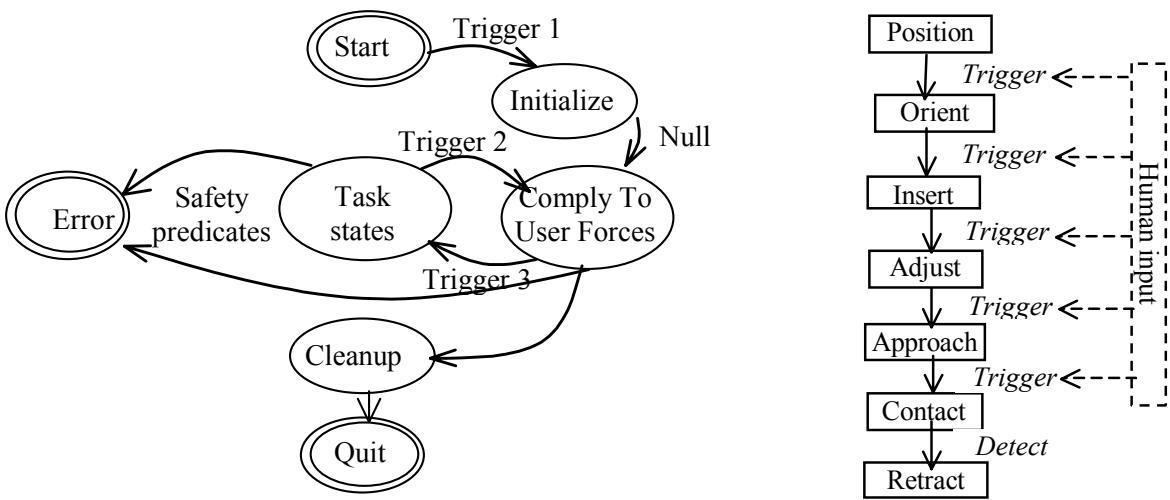

Fig. 1: Left: A Simple System Graph. System States are composed of basic states (initalize, cleanup, manipulate, error) and task specific states. Triggers Between task specific states, and initialization are user interface actions performed by the user. Data Collection is not shown. Right: Task graph for the example task, triggers between states are provided by user by pressing a foot pedal

Primitives are the next level entities. They are composed from sensing and manipulation routines. The primary primitives in "steady hand" manipulation are those that support compliant motion. A "move in compliance to forces" primitive is composed of sensing routines to obtain resolve value of the sensor, a control law (e.g. velocity proportional to force error), and manipulation routines that receive the output of the control law.

Actions are composed from primitives by specifying sensing primitives (predicates) that initiate the execution of the action. These predicates form the conditions that must be met before the primitive is executed. In the above example, the actions are composed of a single "move in compliance to forces" primitive with different parameters. Position uses the translation stages of the robot to provide XYZ positioning where as the rest of the actions (orient, insert, adjust, approach, retract) use two rotational degrees of freedom (about X,Y) and Z insertion degree of freedom about a mechanically constrained remote center of motion(RCM). In the example task, the contact action serves only as placeholder for manipulation actions that might follow in a real task. 
The task representation for a given task is obtained by identifying distinct parts in the conventional approach. Given that "steady hand" manipulation imposes a sequential ordering on planned task execution (user only performs one action at a time with the manipulator), each of these parts can be implemented as an action. The conditions that need to be met before each action are identified. They form the predicates for that action. If an action is composed of several primitives, the process of identifying primitives is repeated for the action. Finally the user identifies safety requirements, such as limits on motion, sensory values etc for each action. This serves as a skeleton for the task graph. The task graph is then executed in a training enviornment. During execution the user may identify redundant or additional states, predicates that modify the task graph.

The system maintains a basic set of states, and predicates. These include initialization and cleanup, a manipulation set, data collection set, and safety and error checking predicates. The system basic states are sleep, and move in compliance to forces, and error states. The data collection set includes a single Dump state. Safety Predicates include workspace and force limits, and hardware and software errors.

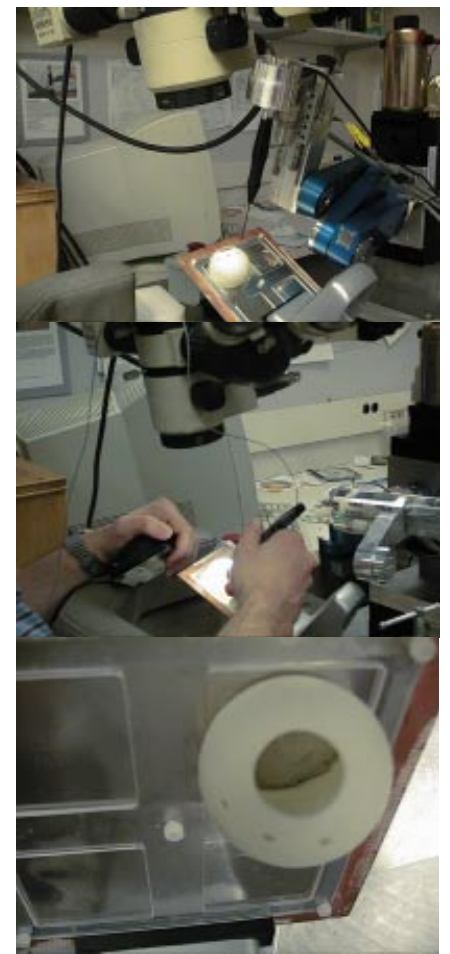

Fig. 2: Experimental Setup: setup with the robot (top), a user performing free hand experiment (middle), and the ball with access ports and data grid (bottom)

\section{Preliminary Experiments}

We have begun to experimental studies to evaluate the effectiveness of using taskgraph enhanced "steady hand" augmentation in comparison to simpler augmentation and un-augmented free hand performance. The task chosen is a constrained needle placement task that presents many of the fine manipulation difficulties encountered in eye surgery. The example task is a modified (or minimally invasive) version of the peg-in-hole task, a common task used for performance evaluation [24],[25],[26].

\subsection{Experimental Environment}

The experimental environment [7] consists of software and hardware components. The software consists of the machine level robot control software and the framework specific to this work. The JHU Modular Robot Control (MRC) library provides the machine level robot control functionality. The hardware consists a cooperative manipu- 
lator and augmented tools required for selected tasks. The cooperative manipulator used is the "steady hand" robot. This is a 7-degree-of-freedom manipulator with XYZ translation at the base for coarse positioning, two rotational degrees of freedom at the shoulder (the RCM linkage, [36]), and instrument insertion and rotation stages. A force sensor is built in the end-effector. This robot has a remote center of motion and an overall positional accuracy of less than 10 micrometers.

This experimental setup appears in Figure 2. To construct the target a ball was sliced and ports constructed to reflect distances similar to the eye. This ball was attached to a data surface containing 100 micrometer holes separated by $2 \mathrm{~mm}$. An ergonomic tool handle was mounted with a $1 \mathrm{~mm}$ shaft and 50 micrometer tip wire for the tool. The goal of the experiment is to touch the bottom of the hole inside the "eye" without touching the sides. Automatic electrical contact sensing is employed to detect contact between the bottom of the holes (success) or the sides of the hole or elsewhere on the surface of the data surface (error).

\subsection{Task and Experimental Protocol}

The selected task can be performed free hand (Unassisted) and with the "steady hand" robot. Two sets of augmentation parameters can be used, one using just constant gain compliant motion (Comply), and another using a non-linear gain scheme (Augmented). With non-linear gains (Figure 3), the gains for each action are modified as a function of distance to the target for that action (since the actions are positioning/orientation primitives, this predicate is easy to evaluate). The target for coarse manipulation actions is the center of port on the ball, and for fine manipulation a selected hole. The port and hole locations are taught to the robot by hand guiding it

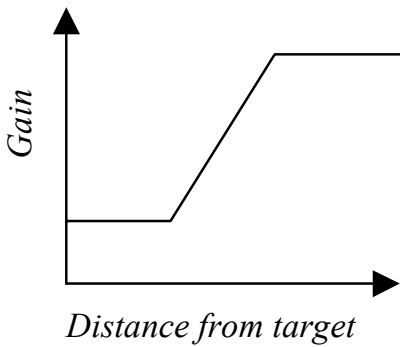

Fig. 3: A typical non- linear gain profile, the target is at zero. to both locations.

The users are allowed unlimited training time till they are comfortable with the experimental protocol, both free hand and with the robot. The users execute each task 5 times. For both robotic and free hand experiments transitions are explicitly signaled by the user/observer (by pressing a button/pedal). Each user also evaluates the setup subjectively on ease of operation, seating comfort, and ease of viewing the target.

\subsection{Results}

From the current data, the success rates for this experiment (number of errors per successful try) improve significantly with the robot, and augmentation adds to the improvement. The total time for the task also decreases when the robot is used. Data from three users appears as a graph below. User 1 had the maximum training time and experience with the system, and user 2 and 3 are familiar with the system. Training 
time clearly affects the performance, and further experimentation is needed for analysis and evaluation. These experiments are scheduled.

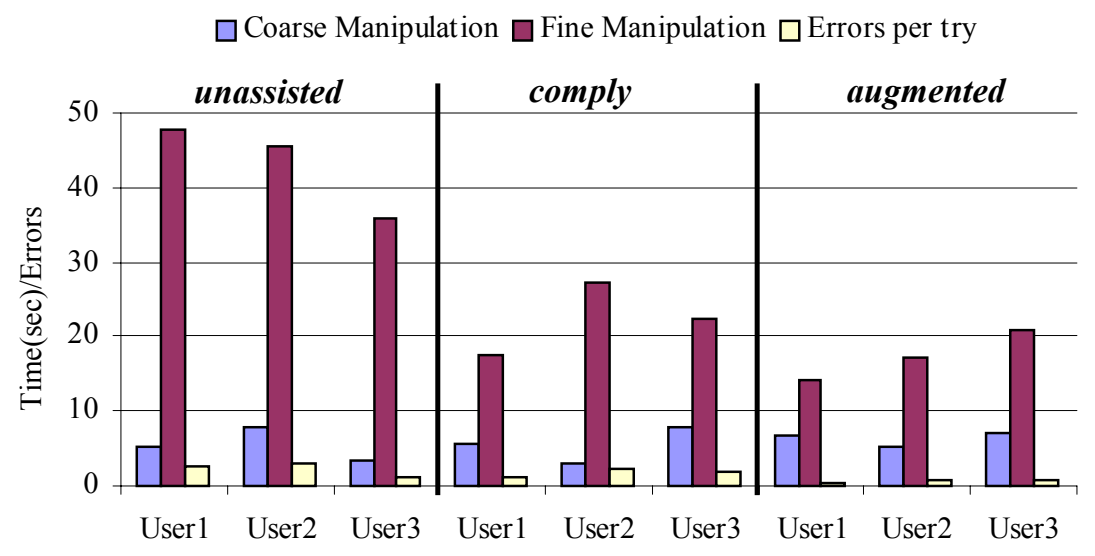

Fig 4: Average Times and errors for the experiment: (Unassisted left, comply center, and augmented right three sets). Errors and total time for the experiment decrease significantly with the robot, and further when non-linear gains are used. The time for coarse motion does not change significantly.

Users find the experiment challenging free hand. The seating conditions are reported as comfortable, and visibility of the target good. The use of foot pedal to determine state transitions was considered non-intuitive, but not difficult by the users. This was replaced with an observer pressing the button at the request of the user. The tip wire would bend and touch the side (when touching the bottom) of the hole with very small forces on it. This may have inflated errors. While this is a problem with all flexible instruments, we are working on improved apparatus and experimental protocol to reduce/detect this problem.

\section{Discussion and Conclusions}

Our system utilizes the sequential nature of the execution of augmented tasks. Note that since this manipulation uses the "steady hand" approach, this is inherent. The example task chosen above is very similar to several other common tasks in surgery, one example being placing a micropipette in a blood vessel to deliver clot-dissolving therapy. However, experimentation is needed with skilled users in conditions even closer to clinical conditions to validate any results. These experiments are also planned.

We do not describe the mechanisms for specification of tasks here. The user describes the task in a high level specification being developed. The detailed discussion is beyond the scope of this paper. Use of a foot pedal was found non-intuitive by the users. However, clinicians routinely use foot pedals and their response to the pedal 
may be different. Alternative mechanisms for state transitions (pause in motion, automatic detection by comparison of change in state <force,position, velocity> ) are available but they are yet to be evaluated.

\section{Summary}

We report on a sensor driven system for augmentation based on "steady hand" manipulation. Under this approach, surgical procedures are performed by a surgeon in the same way as the convention approach. However, portions of the procedure are augmented/automated at surgeon's initiation. The system is described in detail and an implementation is discussed. A preliminary experiment and some preliminary experimental results for an example task are reported.

\section{Acknowledgements}

The authors gratefully acknowledge the support of the National Science Foundation under grant \#IIS9801684, and the Engineering Research Center grant \#EEC9731478. This work was also supported in part by the Johns Hopkins University internal funds.

\section{References}

[1] D. J. Glencross, "Control of skilled movement," Psychological Bulletin, vol. 84, pp. 14-19, 1977.

[2] M. Patkin, "Ergonomics applied to the practice of microsurgery.," Aust NZ J Surg, vol. 47, pp. 320-329, 1977.

[3] K. R. Boff, L. Kaufman, and J. P. Thomas, Handbook of perception and human performance. New York, NY: John Wiley and Sons, 1986.

[4] K. R. Boff and J. E. Lincoln, Engineering Data Compendium: Human Perception and Performance. Ohio: H. G. Anderson Aerospace Medical Research Laboratory., 1988.

[5] R. D. Howe, W. J. Peine, D. A. Kontarinis, and J. S. Son, "Remote palpation technology.," IEEE Engineering in Medicine and Biology, vol. 14, pp. 318323, 1995.

[6] D. A. Kontarinis and R. D. Howe, "Tactile Display of Vibratory Information in Teleoperation and Virtual Environments," Presence, vol. 4, pp. 387-402, 1995.

[7] R. H. Taylor, P. Jensen, L. L. Whitcomb, A. Barnes, R. Kumar, D. Stoianovici, P. Gupta, Z. Wang, E. d. Juan, and L. Kavoussi, " A Steady-Hand Robotic System for Microsurgical Augmentation," presented at Medical Image Computing and Computer-Assisted Intervention - MICCAI'99, Cambridge, UK, 1999. 
[8] T. Lozano-Perez, M. T. Mason, and R. H. Taylor, "Automatic synthesis of fine-motion strategies for robots," The International Journal of Robotics Research, vol. 3, pp. 3--23, 1984.

[9] A. C. Sanderson and G. Perry, "Sensor Based Robotic Assembly Systems: Research And Applications in Electronic Manufacturing," Proceedings of the IEEE, vol. 71, pp. 856-871, 1983.

[10] R. Taylor, J. Korein, G. Maier, and L. Durfee, "A General Purpose Architecture for Programmable Automation Research," presented at Third Int. Symposium on Robotics Research, Chantilly, 1985.

[11] R. H. Taylor, "The Synthesis of Manipulator Control Programs from Task Level Specifications," : Stanford University, 1976.

[12] H. Asada and H. Izumi, "Teaching and Program Generation for the Hybrid Position/Force Control via the Measurement of Human Manipulation Tasks," J. of the Robotics Society of Japan, vol. 5, pp. 452-459, 1987.

[13] H. Asada and B.-H. Yang, "Skill Acquisition from Human Experts through Pattern processing of teaching data," Jornal of Robotics Society of Japan, vol. 8, pp. 17-24, 1990.

[14] K. Kosuge, Y. Fujisava, and T. Fukuda, "Control of Robot Directly Maneuvered by Operator," presented at IEEE International Conference on Robots and Systems, 1993.

[15] K. Kosuge, H. Yoshida, and T. Fukuda, "Dynamic Control for Robot-Human Collaboration," presented at IEEE International Workshop on Robot Human Cooperation, 1993.

[16] K. Kosuge and N. Kazamura, "Control of a robot handling an object in cooperation with a human," presented at IEEE international workshop on Robot human Communication, 1997.

[17] H. Kazerooni, "Human/robot interaction via the transfer of power and information signals --- part i: Dynamics and control analysis," presented at Proceedings of IEEE International Conference on Robotics and Automation, 1989.

[18] H. Kazerooni, "Human/robot interaction via the transfer of power and information signals --- part ii: Dynamics and control analysis," presented at Proceedings of IEEE International Conference on Robotics and Automation, 1989.

[19] H. Kazerooni and G. Jenhwa, "Human extenders," Transaction of the ASME: Journal of Dynamic Systems, Measurement and Control, vol. 115, pp. 21890, June, 1993.

[20] E. A. Dickmanns, "Dynamic Vision Merging Control Engineering and AI Methods," in The Confluence of Vision and Control, G. D. H. e. al, Ed.: Springer-Verlag, pp. 210-229.

[21] S. Lee and S. Ro, "Robotics with Perception and Action Nets," in Control in Robotics and Automation: Sensor Based Integration, N. X. B K Ghosh, T. J. Tarn, Ed. San Diego, CA: Academic Press, 1999, pp. 347-380.

[22] B. L. Davies, K. L. Fan, R. D. Hibberd, M. Jakopec, and S. J. Harris, "ACROBOT - Using Robots and Surgeons Synergistically in Knee Surgery," presented at 8th International Conference on Advanced Robotics, California, USA, 1997. 
[23] J. Troccaz, M. Peshkin, and B. L. Davies, "The use of localizers, robots, and synergistic devices in CAS," presented at Proc. First Joint Conference of CVRMed and MRCAS, Grenoble, France, 1997.

[24] R. Kumar, T. Goradia, A. Barnes, P. Jensen, L. Whitcomb, D. Stoianovici, L. Auer, and R. Taylor, "Performance of Robotic Augmentation in Microsurgery-Scale Motions," presented at 2nd Int. Symposium on Medical Image Computing and Computer-Assisted Surgery, Cambridge, England, 1999.

[25] S. E. Salcudean, S. Ku, and G. Bell, "Performance measurement in scaled teleoperation for microsurgery," presented at First joint conference computer vision, virtual realtiy and robotics in medicine and medical robotics and computer-assisted surgery, Grenoble, France, 1997.

[26] H. Das, H. Zak, J. Johnson, J. Crouch, and D. Frambach, "Evaluation of a Telerobotic System to Assist Surgeons in Microsurgery," Computer Aided Surgery, vol. 4, pp. 15-25, 1999. 